\title{
Biomimetic Magnetic Nanocomposite for Smart Skins
}

\author{
A. Alfadhel', J. Kosel \\ Computer, Electrical and Mathematical Sciences and Engineering Division (CEMSE) \\ King Abdullah University of Science and Technology (KAUST) \\ Thuwal, Saudi Arabia \\ *Email: ahmed.fadhel@kaust.edu.sa
}

\begin{abstract}
We report a biomimetic tactile sensor consisting of magnetic nanocomposite artificial cilia and magnetic sensors. The nanocomposite is fashioned from polydimethylsiloxane and iron nanowires and exhibits a permanent magnetic behavior. This enables remote operation without an additional magnetic field to magnetize the nanowires, which simplifies device integration. Moreover, the highly elastic and easy patternable nanocomposite is corrosion resistant and thermally stable. The highly sensitive and power efficient tactile sensors can detect vertical and shear forces from interactions with objects. The sensors can operate in dry and wet environment with the ability to measure different properties such as the texture and the movement or stability of objects, with easily adjustable performance.
\end{abstract}

Keywords - Smart Skin, Nanocomposite, Nanowires, Magnetic, Cilia, Tactile sensor

\section{INTRODUCTION}

Evolutions in robotics or prosthetics are demanding an increased perception of the environment including touch. Noticeable progress in the field of artificial skins lead to the development of different technologies that can mimic the complex sense of touch in humans for better interaction with the surrounding environment. These skins are also important for applications like health monitoring systems and smart surgical tools. Tactile sensors are the essential components in the artificial skin development that attracted considerable attention and resulted in introducing different sensing mechanisms [1-3]. However, many technical challenges still remain in current technologies, including the limited performance and the high power consumption, in addition to the interference with environmental noise.

Artificial cilia sensors became attractive for researchers to mimic the extremely sensitive microscale cilia receptors [4-6]. The high aspect ratio and surface area to volume ratio of cilia allow good interaction with the environment.

Advances in material integration and nanofabrication technologies have enabled new directions of research and development for micro-sensor systems. Examples are nanocomposite materials, which combine the advantages of polymers like flexibility, elasticity, chemical resistance or biocompatibility with the unique properties of additives [6-8]. Magnetic nanocomposites have been introduced before using superparamagnetic particles $[9,10]$. Unlike these particles that require a high magnetic field for magnetization, which increases the power consumption and limits the possibility of integration and miniaturization, iron nanowires (NWs) have a high magnetization at remanence due to the strong shape anisotropy, and they can be easily fabricated with a cost effective process. Combining these NWs with a polymer results in a magnetic nanocomposite with distinct features.

In our work, we realize a magnetic artificial cilia array integrated on a magnetic sensing element for tactile sensing applications. The cilia are fabricated from a nanocomposite that is made of iron NWs incorporated into polydimethylsiloxane (PDMS). The developed nanocomposite utilizes the permanent magnetic behavior of the NWs, is highly elastic, can easily be patterned and has a good corrosion resistance.

\section{METHODS}

\section{A. Sensor Concept}

The operating principle of the sensor is based on detecting the change of the stray magnetic field, created by the permanent magnetic cilia, when deflected by an external force (Fig. 1). A giant magneto-impedance (GMI) magnetic field sensor, which offers a high sensitivity, is simple to fabricate and can be realized on flexible substrates, is utilized to measure the change in the magnetic field [11-15]. At small bias fields and at high operating frequencies, the impedance of GMI sensors is sensitive to external magnetic fields, due to the strong dependence of the skin effect on the magnetic permeability.
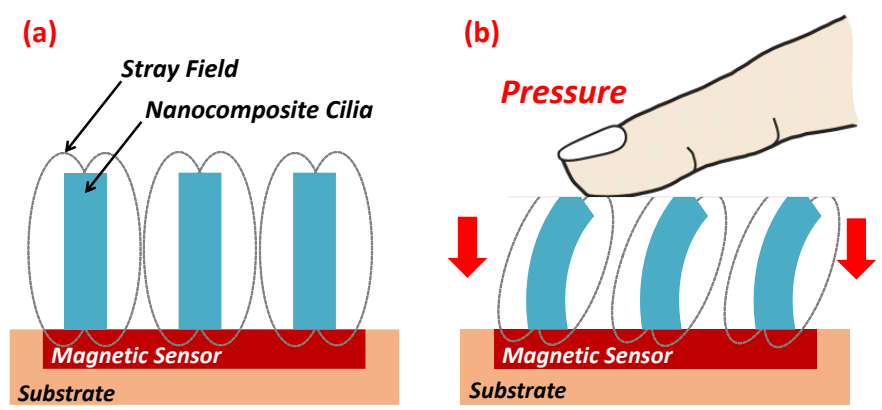

Fig. 1: (a) The working principle of the tactile sensor. (b) When the cilia are deflected by applying pressure, the stray field measured with the giant magnetoimpedance (GMI) sensor changes, changing the impedance. 
A distinct advantage of the cilia is the permanent magnetic behavior of the iron NWs, eliminating the need for an external magnetic field to magnetize the cilia or bias the GMI sensor. At resting position, the stray field of the cilia affects the GMI sensor with an average magnetic field value that biases the sensor and changes its initial impedance. In the presence of an external force, the cilia bend in the force direction. This bending results in a change of the stray field of the cilia affecting the GMI sensor, hence changing the sensor's impedance.

\section{B. Fabrication}

Iron NWs with $6 \mu \mathrm{m}$ in length and $35 \mathrm{~nm}$ in diameter are fabricated by the electrodeposition into a nanoporous aluminum oxide membrane, prepared by a two-step anodization process using oxalic acid on an aluminum substrate. The magnetic nanocomposite is prepared by mixing NWs dispersed in Sodium dodecyl sulfate (SDS) surfactant with PDMS (Sylgard 184 Silicone Elastomer, Dow Corning Corporation) that is used as the polymeric matrix. The nanocomposite is then cured at $90^{\circ} \mathrm{C}$ for 1 hour (Fig. 2). This process allows adjusting the NWs concentration easily. The NWs can also be aligned in the nanocomposite by applying a homogenous magnetic field along the desired direction during the curing process.

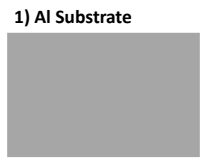

5) NWs Electrodeposition

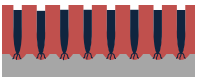

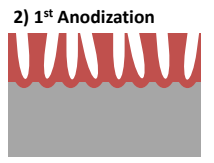

6) NWs Release

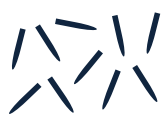

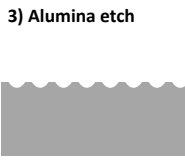

4) $2^{\text {nd }}$ Anodization + Barrier reduction

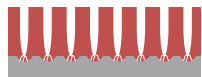

7) NWs mixing with polymer

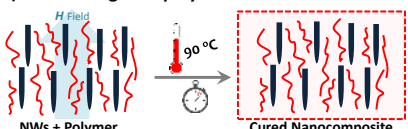

Fig. 2. Illustration of the electrochemical fabrication process of iron NWs and the process of fabricating the nanocomposite. Aluminum substrate is used in the process (1) and two anodization steps are performed using oxalic acid to obtain uniform nanopores of $\sim 30 \mathrm{~nm}$ in diameter, followed by a barrier reduction step for establishing an electrical contact with the bottom electrode (2-4). Iron is electrodeposited into the nanopores (5) and then the NWs are released by etching the Alumina membrane and dispersed in ethanol (6). The nanocomposite is fabricated by mixing the NWs with the polymer and then curing the composite. The NWs can be aligned in a certain direction by applying an external homogenous magnetic field during the curing process (7).

A three-layer GMI magnetic field sensor that has a meander structure of $2 \times 2 \mathrm{~mm}^{2}$ and has $2 \mathrm{~mm}$ long and $200 \mu \mathrm{m}$ wide conductors with $200 \mu \mathrm{m}$ spacing is fabricated to detect the change of the stray magnetic field. The GMI sensor is composed of $200 \mathrm{~nm}$ thick $\mathrm{Cu}$ layer sandwiched by two 100 nm thick $\mathrm{Ni}_{80} \mathrm{Fe}_{20}$ layers. The GMI sensor structure is patterned onto a substrate using standard lithography and the materials are deposited by e-beam evaporation with a constant magnetic field of 100 Oe applied in the transverse direction to induce a uniaxial magnetic anisotropy, creating a magnetically sensitive axis in the longitudinal direction, and then followed by a lift-off process.

The nanocomposite has 20\% NWs:PDMS volume ratio. The NW ratio in the nanocomposite is chosen to provide the biasing field for the GMI sensor and, at the same time, to not adversely affect the polymerization process of the PDMS or the elasticity of the cilia. Nanocomposite cilia are fabricated using a master mold technique. A $1 \mathrm{~mm}$ thick poly(methylmethcrylate) (PMMA) master mold into which an array of holes with $200 \mu \mathrm{m}$ in diameter is patterned with a $\mathrm{CO}_{2}$ laser cutter (Universal PLS6.75). The nanocomposite is casted onto the surface of the GMI sensor and the master mold is mounted on top with aligning the holes along the GMI sensor using a microscope. This structure is then placed in a desiccator for 30 minutes to remove any trapped air bubbles and assist in filling the pores. Next, the composite is cured at 90 degrees Celsius for one hour with external magnetic field along the length of the pillar, forming the cilia with aligned NWs on top of the GMI sensor. After releasing the cured cilia, they are fully magnetized by applying a magnetic field of 10 kOe. The cilia are arranged on the GMI sensor such that they can almost fully deflect and are placed along conductors with the same current direction. The fabrication process is illustrated in Fig. 3. (a)

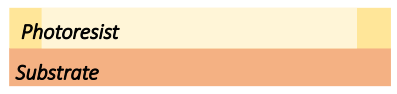

(c)

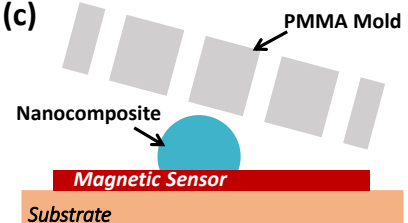

(e)

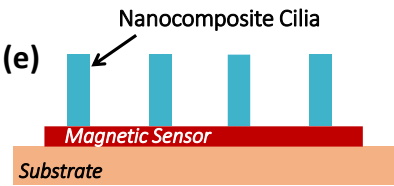

(b)

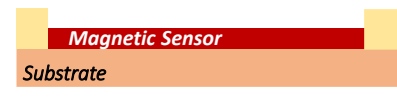

(d)

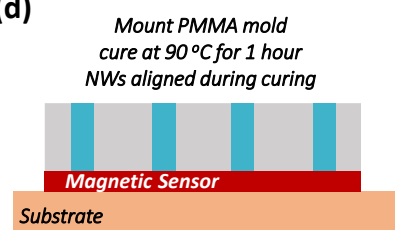

$500 \mu \mathrm{m}$

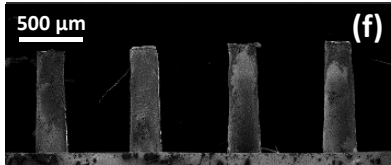

Fig. 3. Fabrication process of the magnetic cilia tactile sensor. (a,b) A GMI sensor is fabricated by depositing $100 \mathrm{~nm}$ permalloy/ $200 \mathrm{~nm} \mathrm{Cu} / 100 \mathrm{~nm}$ permalloy by e-beam evaporation and it is patterned using a standard lithography process. (b-e) Cilia are fabricated using a PMMA mold. (f) Cilia with $1 \mathrm{~mm}$ in length and $200 \mu \mathrm{m}$ in diameter.

\section{Characterization}

The magnetization curves along the length of the cilia and along their perpendicular direction are obtained using a vibrating sample magnetometer. As shown in Fig. 4, the cilia with vertically aligned NWs have a magnetic anisotropy with a remanence to saturation magnetization of $95 \%$ along the vertical direction with a coercivity of $1.5 \mathrm{kOe}$, and magnetization of $37 \%$ along the horizontal direction. These results show the permanent magnetic like behavior of the nanocomposite and confirm the alignment of the NWs.

The GMI sensor is tested by applying a magnetic field with a Helmholtz coil and measuring the impedance with an impedance analyzer (Agilent E4991A) at a current of $0.1 \mathrm{~mA}$ in amplitude. The results in Fig. 5 show that the GMI sensor has higher sensitivity at higher frequencies as expected [15]. At $500 \mathrm{MHz}$, the GMI sensor response shows a peak at a field of 10 Oe corresponding to the anisotropy field of the $\mathrm{Ni}_{80} \mathrm{Fe}_{20}$ thin film, and it has a maximum sensitivity of $1.2 \Omega / \mathrm{Oe}$. 


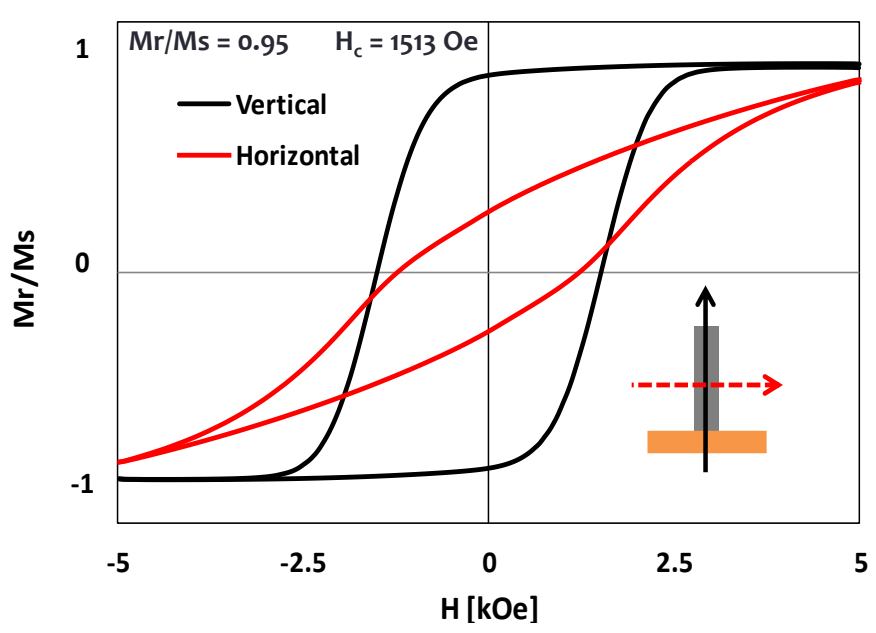

Fig. 4. Nanocomposite magnetization curves obtained with a vibrating sample magnetometer in the vertical and horizontal direction for vertically aligned NWs.

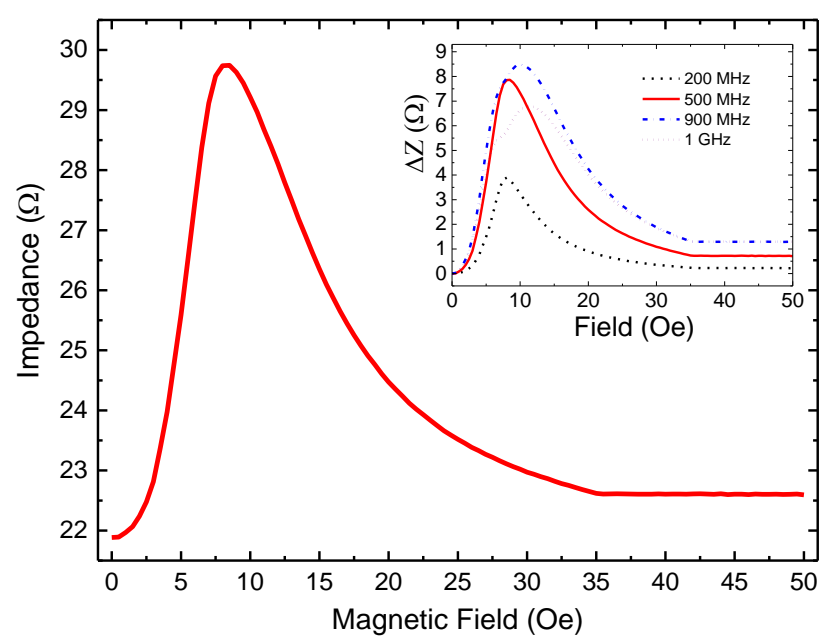

Fig. 5. Impedance response of the GMI sensor at $1 \mathrm{~mA}$ and $500 \mathrm{MHz}$. Inset: GMI sensor response at different frequencies.

\section{RESULTS}

The performance of the tactile sensor is tested for $1 \mathrm{~mm}$ long and $200 \mu \mathrm{m}$ in diameter cilia arrays on $4 \mathrm{~mm}^{2}$ magnetic meander sensors. Arrays with 9 and 24 cilia are investigated. The sensor is characterized by applying vertical forces using a stepper motor and collecting the impedance response with an impedance analyzer. As shown in Fig. 6, the sensor with 9 cilia can detect vertical forces up to $200 \mathrm{mN}(50 \mathrm{kPa})$ with a resolution of $0.9 \mathrm{mN}(0.23 \mathrm{kPa})$ and a sensitivity of 15 $\mathrm{m} \Omega / \mathrm{mN}(60 \mathrm{~m} \Omega / \mathrm{kPa})$. The 24 cilia arrangement shows a response up to $680 \mathrm{mN}(170 \mathrm{kPa})$ with a resolution of $3.5 \mathrm{mN}$ $(0.88 \mathrm{kPa})$ and a sensitivity of $4 \mathrm{~m} \Omega / \mathrm{mN}(16 \mathrm{~m} \Omega / \mathrm{kPa})$. The sensor operates at an extremely low power consumption of 80 $\mathrm{nW}$. The results are compared to a reference measurement for cilia without NWs to obtain the effect of pressure on the GMI sensor and the effect of the test setup on. Almost no response was obtained from the reference measurement.

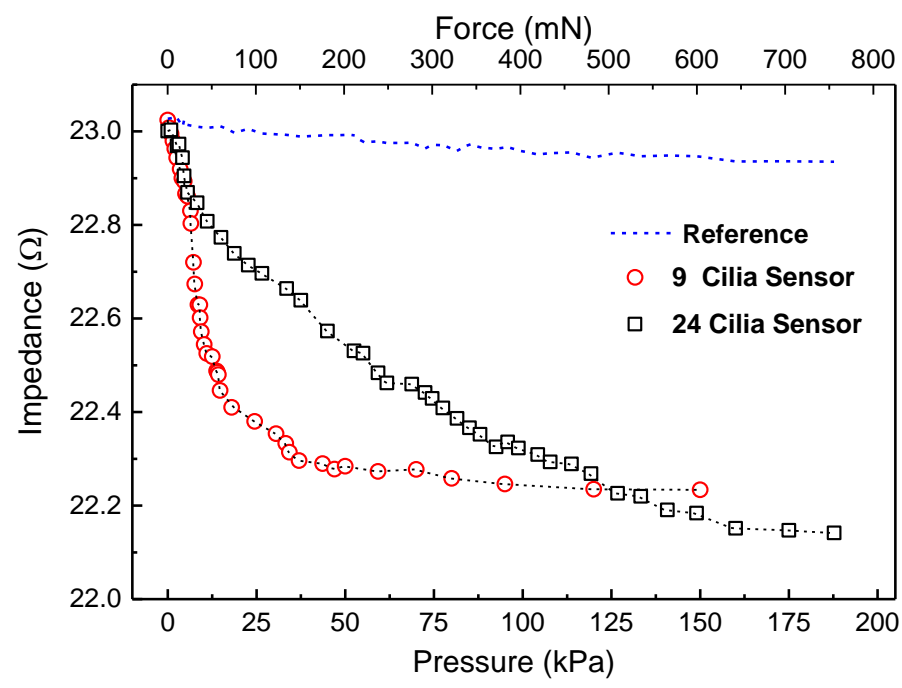

Fig. 6. Tactile sensor impedance response for two cilia configurations: 9 and 24 cilia array, compared to a reference measurement.

The tactile sensor can also detect shear forces. Fluid flows have been utilized representing shear forces applied to the cilia for sensors implemented in a microfluidic channel as reported previously [6]. These experiments have shown the ability to detect air flow rates up to $190 \mathrm{~mm} / \mathrm{s}$ with a sensitivity of 24 $\mathrm{m} \Omega /(\mathrm{mm} / \mathrm{s})$ and a resolution of $0.56 \mathrm{~mm} / \mathrm{s}$, while the range for water flow is up to $7.8 \mathrm{~mm} / \mathrm{s}$ with sensitivity of $0.9 \Omega /(\mathrm{mm} / \mathrm{s})$ and a high resolution of $15 \mu \mathrm{m} / \mathrm{s}$.

An advantage of using a highly sensitive magnetic sensor is, the possibility to detect the orientation with respect to the Earth's magnetic field. To distinguish the orientation change from the cilia deflection response, an additional GMI sensor can be used as an element in the tactile sensor. The experiment was conducted by gradually changing the sensor's orientation from the parallel to the perpendicular direction with respect to the horizontal plane. Fig. 7 shows the sensor impedance when rotated through $180^{\circ}$. The impedance changes by a maximum of $50 \mathrm{~m} \Omega$, where the peak value corresponds to the sensor's sensitive direction being aligned parallel with the Earth's magnetic field.

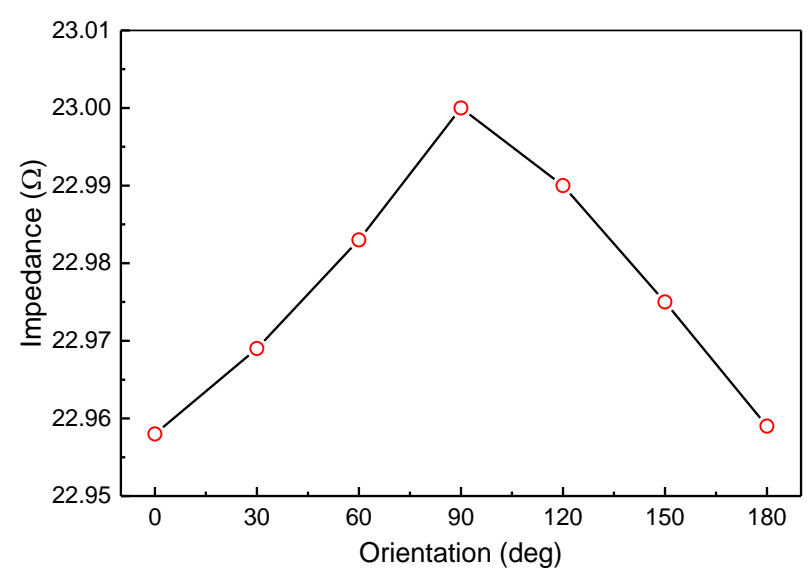

Fig. 7. Tactile sensor's response at different orientations with respect to the earth's magnetic field. 


\section{CONCLUSION}

A novel NWs-based permanent magnetic and highly elastic nanocomposite cilia tactile sensor has been realized for smart skin application. This magnetic based cilia sensor concept utilizes a magnetic sensor to detect the change of the stray field created by the permanent magnetic cilia when deflected by a force, in addition to biasing the GMI sensor at its operation point. This greatly simplifies miniaturization, aids integration and minimizes the power consumption. The magnetic properties of the nanocomposite can be tailored in a wide range by the choice of the materials and the dimensions of the polymer and NWs as well as by the concentration of the NWs. Three-layer GMI sensors are used because they offer a good compromise between sensitivity and fabrication complexity. They can be implemented as wireless devices and on flexible substrates, which can be utilized to realize passive, remote and bendable sensors.

The developed sensor has a high sensitivity and extremely low power consumption, and has a wide range of control over sensing regime, sensitivity and resolution by simply modifying the dimensions of the cilia.

\section{ACKNOWLEDGEMENTS}

Research reported in this publication was supported by the King Abdullah University of Science and Technology (KAUST).

\section{REFERENCES}

[1] S. Mohsin, et al., "A review of tactile sensing technologies in biomedical engineering," Sens. Actuators A, vol. 179, pp. 17, 2012.
[2] K. Takei, et al., "Nanowire active-matrix circuitry for low-voltage macroscale artificial skin," Nature Mat., vol. 9, pp. 821, 2010.

[3] C. Pang, et al., "A flexible and highly sensitive strain-gauge sensor using reversible interlocking of nanofibres," Nature Mat., vol. 11, pp. 795, 2012.

[4] M. Hein, et al., "Fabrication of bio inspired inorganic nanocilia sensors," IEEE Trans. on Magnetics, vol. 49, pp. 191, 2013.

[5] C. Liu, "Micromachined biomimetic artificial haircell sensors," Bioinspir. and Biomim., vol. 2, pp. S162, 2007.

[6] A. Alfadhel, et al., "Magnetic nanocomposite for biomimetic flow sensing," Lab Chip, vol. 14, pp. 4362, 2014.

[7] M. Y. Alnassar, Y. P. Ivanov, and J. Kosel, "Fabrication and Properties of Multiferroic Nanocomposite Films," IEEE Trans. Mag., vol. 51, pp. 2500504, 2015.

[8] M. Y. Alnassar, A. Alfadhel, Y. P. Ivanov, and J. Kosel, "Magnetoelectric Polymer Nanocomposite for Flexible Electronics," Journal of Applied Physics, vol. 117, pp. 17D711, 2015.

[9] M. Suter, et al., "A photopatternable superparamagnetic nanocomposite: Material characterization and fabrication of microstructures," Sens. and Actuat. B, vol. 156, pp. 433, 2011.

[10] B. Zhou, W. Xu, A. Syed, Y. Chau, L. Chen, B. Chew, O. Yassine, X. Wu,Y. Gao, J. Zhang, X. Xiao, J .Kosel, X. Zhang, Z. Yao, W Wen," Design and fabrication of magnetically functionalized flexible micropillar arrays for rapid and reversible microfluidic mixing," Lab Chip, DOI: 10.1039/C5LC00173K, 2015.

[11] L. Panina, and K. Mohri, "Magneto-impedance in multilayer films," Sensors Actuators A, vol. 81, pp. 71, 2000.

[12] B. Li, et al., "Integration of thin film giant magneto impedance sensor and surface acoustic wave transponder," J. Appl. Phys., vol. 111, pp. 07E514, 2012.

[13] B. Li, and J. Kosel, "Three dimensional simulation of giant magnetoimpedance effect in thin film based sensors," Journal of Applied Physics, 109, pp. 07E519, 2011.

[14] B. Li, A. Morsy, and J. Kosel, "Optimization of autonomous magnetic field sensor consisting of giant magnetoimpedance sensor and surface acoustic wave transducer," IEEE Trans. Magnetics, 48, vol. 11, pp. 4324, 2012.

[15] B. Li, M. N. Kavaldzhiev, J. Kosel, "Flexible magnetoimpedance sensor," J. of Mag. and Mag. Mater., vol. 378, pp. 499, 2015. 